\title{
Clinical implications of the cortical hyperintensity belt sign in fluid-attenuated inversion recovery images after bypass surgery for moyamoya disease
}

\author{
Eika Hamano, MD, ${ }^{1}$ Hiroharu Kataoka, MD, PhD, ${ }^{1}$ Naomi Morita, MD, PhD, ${ }^{2}$ \\ Daisuke Maruyama, MD, ${ }^{1}$ Tetsu Satow, MD, PhD, ${ }^{1}$ Koji lihara, MD, PhD, ${ }^{1}$ and \\ Jun C. Takahashi, MD, PhD'1 \\ Departments of ${ }^{1}$ Neurosurgery and ${ }^{2}$ Radiology, National Cerebral and Cardiovascular Center, Suita, Osaka, Japan
}

OBJECTIVE Transient neurological symptoms are frequently observed during the early postoperative period after direct bypass surgery for moyamoya disease. Abnormal signal changes in the cerebral cortex can be seen in postoperative MR images. The purpose of this study was to reveal the radiological features of the "cortical hyperintensity belt (CHB) sign" in postoperative FLAIR images and to verify its relationship to transient neurological events (TNEs) and regional cerebral blood flow ( $\mathrm{rCBF})$.

METHODS A total of 141 hemispheres in 107 consecutive patients with moyamoya disease who had undergone direct bypass surgery were analyzed. In all cases, FLAIR images were obtained during postoperative days (PODs) 1-3 and during the chronic period $(3.2 \pm 1.13$ months after surgery). The CHB sign was defined as an intraparenchymal highintensity signal within the cortex of the surgically treated hemisphere with no infarction or hemorrhage present. The territory of the middle cerebral artery was divided into anterior and posterior parts, with the extent of the CHB sign in each part scored as 0 for none; 1 for presence in less than half of the part; and 2 for presence in more than half of the part. The sum of these scores provided the CHB score (0-4). TNEs were defined as reversible neurological deficits detected both objectively and subjectively. The rCBF was measured with SPECT using N-isopropyl-p-[23|]]iodoamphetamine before surgery and during PODs $1-3$. The $\mathrm{rCBF}$ increase ratio was calculated by comparing the pre- and postoperative count activity.

RESULTS Cortical hyperintensity belt signs were detected in 112 cases (79.4\%) and all disappeared during the chronic period. Although all bypass grafts were anastomosed to the anterior part of the middle cerebral artery territory, CHB signs were much more pronounced in the posterior part $(p<0.0001)$. TNEs were observed in 86 cases $(61.0 \%)$. Patients with TNEs showed significantly higher CHB scores than those without $(2.31 \pm 0.13$ vs $1.24 \pm 0.16, p<0.0001)$. The CHB score, on the other hand, showed no relationship with the rCBF increase ratio $(p=0.775)$. In addition, the rCBF increase ratio did not differ between those patients with TNEs and those without (1.15 \pm 0.033 vs $1.16 \pm 0.037, p=0.978)$.

CONCLUSIONS The findings strongly suggest that the presence of the CHB sign during PODs $1-3$ can be a predictor of TNEs after bypass surgery for moyamoya disease. On the other hand, presence of this sign appears to have no direct relationship with the postoperative local hyperperfusion phenomenon. Vasogenic edema can be hypothesized as the pathophysiology of the CHB sign, because the sign was transient and never accompanied by infarction in the present series.

http://thejns.org/doi/abs/10.3171/2015.10.JNS151022

KEY WORDS moyamoya disease; cortical hyperintensity belt sign; transient neurological events; hyperperfusion; extracranial-intracranial bypass; vascular disorders

ABBREVIATIONS $\mathrm{CHB}=$ cortical hyperintensity belt; $\mathrm{DWI}$ = diffusion-weighted image; $I M P=$ iodoamphetamine; $M C A=$ middle cerebral artery; $P O D=$ postoperative day; PRES = posterior reversible encephalopathy syndrome; rCBF = regional cerebral blood flow; STA = superficial temporal artery; TNE = transient neurological event. SUBMITTED May 3, 2015. ACCEPTED October 22, 2015.

INCLUDE WHEN CITING Published online February 19, 2016; DOI: 10.3171/2015.10.JNS151022. 
$\mathrm{E}$ XTRACRANIAL-INTRACRANIAL direct anastomotic bypass is an established treatment for ischemic moyamoya disease. ${ }^{18,28}$ During the acute period after bypass surgery, however, transient neurological events (TNEs) can be observed despite the hemodynamic improvement. ${ }^{9}$ Local cortical hyperperfusion has been suggested as a cause of these TNEs; $4,5,7,10,23,38$ however, the physiological mechanism of TNEs remains unclear. Recently, a hyperintensity sign observed in FLAIR MRI after bypass surgery has been reported and its correlation with hyperperfusion has been discussed, ${ }^{11}$ but the clinical implications of such radiological findings remain in question. In the present study, we focused on the intraparenchymal "cortical hyperintensity belt (CHB) sign." Its distinctness, location, and correlation with TNEs are examined, and whether this sign can be considered a predictor of postoperative TNEs is discussed.

\section{Methods}

\section{Patient Demographic Data}

This study included 141 hemispheres of 107 consecutive patients with moyamoya disease who had undergone superficial temporal artery-middle cerebral artery (STAMCA) direct anastomosis between April 2009 and August 2014. Eighty-five patients were adult or adolescent $(\geq 15$ years old) and 22 were children ( $<15$ years old). Patient demographic data are listed in Table 1. Cases involving perioperative cortical infarction (5 other cases during the above period) were excluded because the abnormal hyperintensity signal on which this study focused could not be clearly distinguished because of the cortical infarction.

\section{Surgical Procedure}

In all cases, the STA was anastomosed to the cortical MCA $\left(\mathrm{M}_{4}\right)$ in the frontal operculum with 11-0 nylon under a surgical microscope. In 22 hemispheres of 13 pediatric cases, encephalomyosynangiosis ${ }^{17}$ was added to the direct anastomosis.

TABLE 1. Characteristics of 107 patients with moyamoya disease

\begin{tabular}{cc}
\hline Characteristic & Value \\
\hline Age at surgery, yrs & \\
\hline Mean \pm SD & $33.2 \pm 18.7$ \\
\hline Range & $3-72$ \\
$\geq 15, \mathrm{n}(\%)$ & $85(79.4)$ \\
\hline$<15, \mathrm{n}(\%)$ & $22(20.6)$ \\
\hline Sex, $\mathrm{n}(\%)$ & \\
\hline $\mathrm{M}$ & $51(36.2)$ \\
\hline $\mathrm{F}$ & $90(63.8)$ \\
\hline Operative side, $\mathrm{n}(\%)$ & $70(49.6)$ \\
\hline Rt & $71(50.3)$ \\
\hline Lt & \\
\hline Onset, $\mathrm{n}(\%)$ & $117(83.0)$ \\
\hline Ischemia & $16(11.3)$ \\
\hline Hemorrhage & $8(5.7)$ \\
\hline Other &
\end{tabular}

\section{The CHB Sign in MRI}

In all cases, cerebral CT images, FLAIR images, diffusion-weighted images (DWIs), and MR angiograms were obtained before and after surgery, with either a 3.0$\mathrm{T}$ scanner (Verio, Spectra; Siemens) or a 1.5-T scanner (Vision; Siemens). These studies were performed during postoperative days (PODs) 1-3. The criteria for identifying the CHB sign were as follows: 1) presence of an intraparenchymal high-intensity signal within the cortex or subcortex of the MCA territory of the surgically treated hemisphere; 2) absence of acute infarction; and 3) absence of acute hemorrhage (Fig. 1 left). A high-intensity signal in the cortical sulci, commonly called the "ivy sign" (Fig. 1 right), ${ }^{25,32}$ was strictly excluded from consideration as the CHB sign.

All slices of the axial FLAIR images were reviewed to determine the extent to which the CHB sign was present, according to a procedure adapted from a previous report. ${ }^{30}$ The central sulcus represented the division of the MCA territory between anterior and posterior parts; the temporal lobe was included in the posterior part. In each part, the extent of the CHB sign was scored as 0 (none visible), 1 (limited to within half of the part), or 2 (extending over more than half of the part). The total CHB score was the sum of the local scores (minimum $=0$, maximum $=4)$. The CHB scores were determined through the joint agreement of 2 neurosurgeons (E.H. and H.K.), who were blinded to the neurological condition and outcome of each case. Examples of CHB signs are shown in Fig. 2. In all patients, MRI was also performed during the chronic period ( $3.2 \pm 1.13$ months after surgery).

\section{Neurological Evaluation}

Patients' clinical records were evaluated retrospectively. The criteria for identifying postoperative TNEs were as follows: 1) reversible neurological deficits observed objectively (e.g., motor weakness, aphasia); 2) reversible neurological deficits recognized subjectively and reported by the patients (e.g., numbness of the extremities); 3) no sign of acute cerebral infarction in radiological images; and 4) no sign of acute hemorrhage in radiological images. In each patient, the total number of symptomatic days was recorded and defined as the duration of the postoperative TNEs.
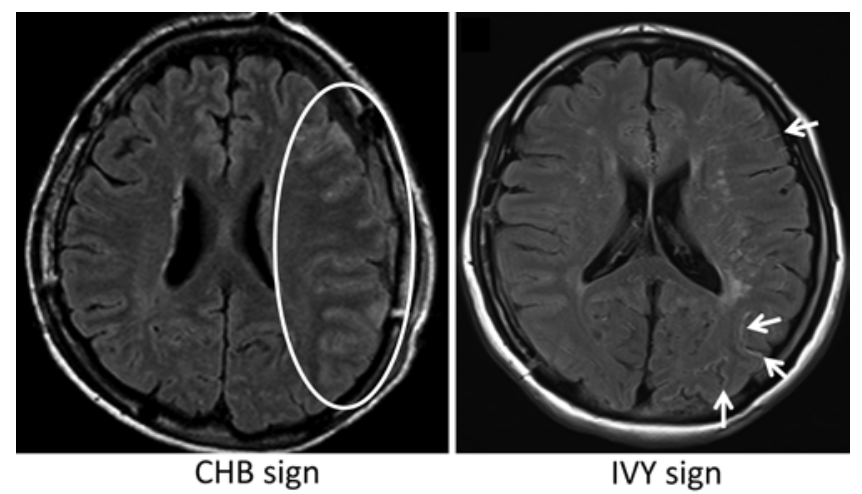

FIG. 1. Differences in MR FLAIR images. Left: The CHB sign (circled). Right: The ivy sign (arrows). 

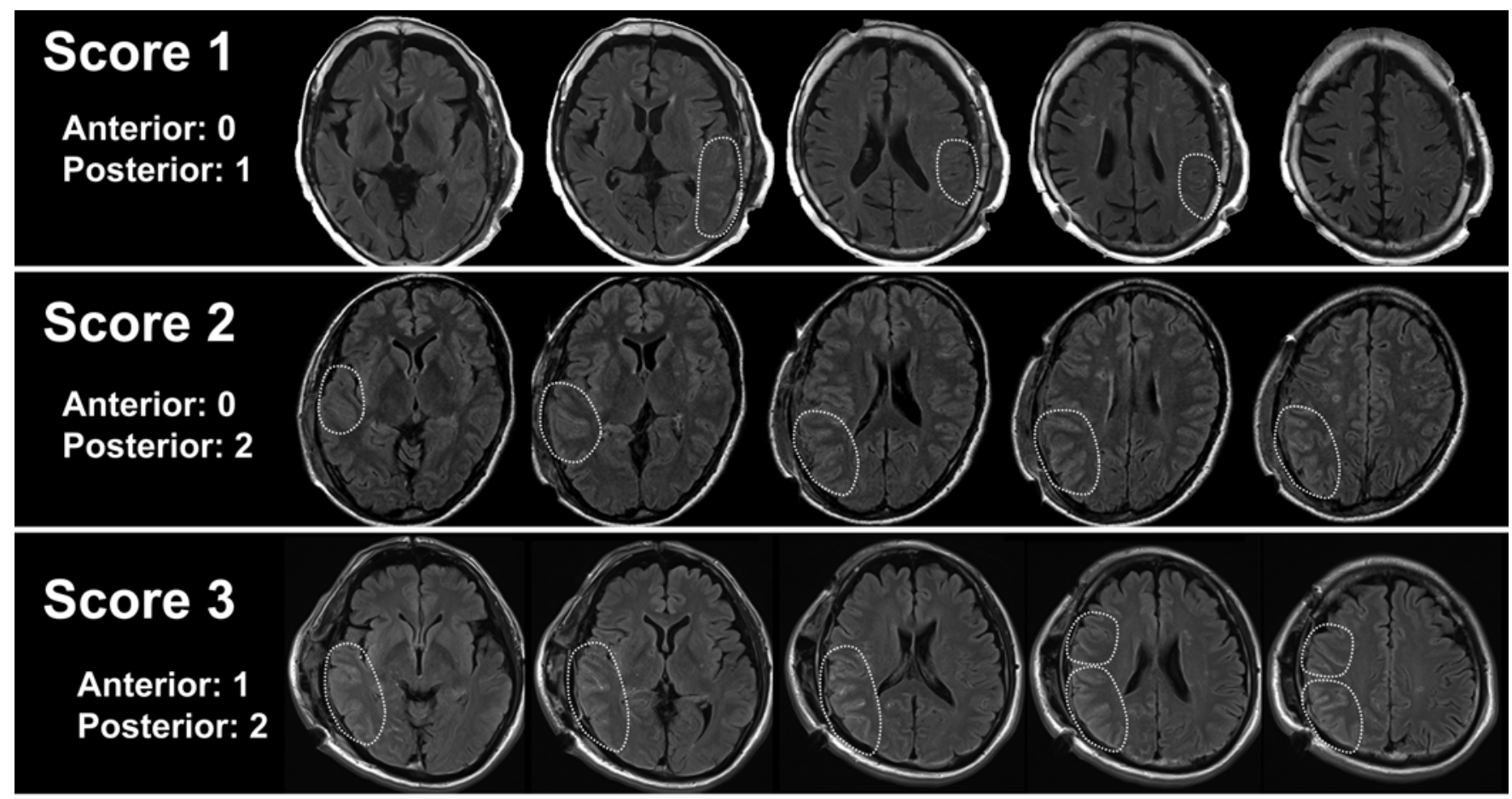

\section{Score 4}

Anterior: 2

Posterior: 2

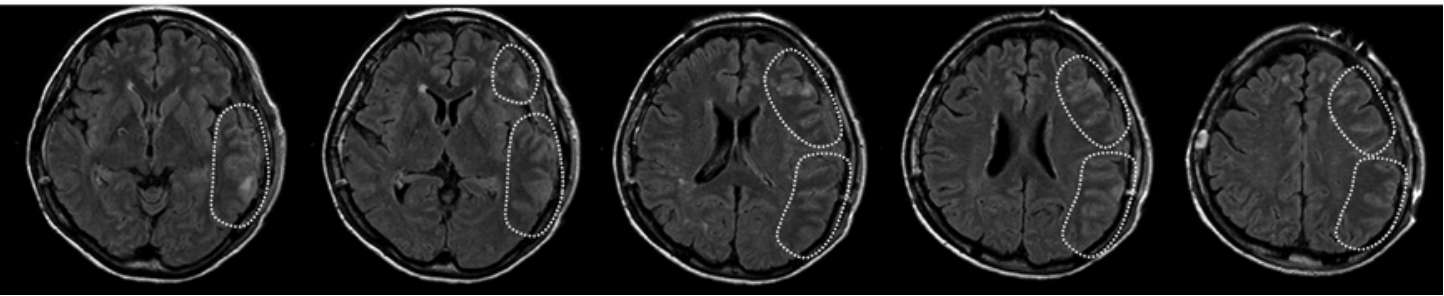

FIG. 2. Examples of the scoring of the CHB sign. The MCA territory in the cortico-subcortical region is divided into anterior and posterior parts in all slices of axial FLAIR images. The anterior and posterior parts are separated by the central sulcus. The temporal lobe is included in the posterior part. In each part, the extent of the CHB sign is scored as 0 (none visible), 1 (limited to within half of the part), or 2 (extending over more than half of the part). The total CHB score is the sum of the local scores (minimum $=0$, maximum $=4$ ).

\section{Cerebral Blood Flow Measurement}

Preoperative regional cerebral blood flow (rCBF) was evaluated with SPECT using N-isopropyl-p-[ $\left.{ }^{[23} I\right]$ iodoamphetamine (123I-IMP) within the month before surgery. In 18 patients, ${ }^{15} \mathrm{O}$-gas PET was used instead of SPECT. In all cases, postoperative ${ }^{123}$ I-IMP SPECT was performed during PODs 1-3. Images were transferred to a workstation and analyzed using Multimodality Image Registration Software (Dr. View; AJS, Inc.). Circular regions of interest measuring $2 \mathrm{~cm}$ in diameter were selected in areas with increased $\mathrm{rCBF}$ and in the ipsilateral cerebellum, and activity was measured. Cerebral-to-cerebellar activity ratios were calculated, and the ratio of postoperative to preoperative cerebral-to-cerebellar ratios was regarded as the "rCBF increase ratio."

\section{Statistical Analysis}

Data are expressed as the mean $\pm \mathrm{SD}$. A Wilcoxon signed-rank test was used to compare the CHB scores of the anterior and posterior parts of the MCA territory. A Mann-Whitney U-test was used to compare the CHB scores of groups with and without TNEs. Spearman's p test was used to calculate the rank correlation coefficient (r) between 1) duration of TNEs and CHB score, and 2) rCBF increase ratio and $\mathrm{CHB}$ score. A 1-way ANOVA was used to compare the $\mathrm{rCBF}$ increase ratios within each $\mathrm{CHB}$ score group. Student's t-test was used to assess the rCBF increase ratio between groups with and without TNEs. Values of $\mathrm{p}<$ 0.05 were considered statistically significant. All analyses were performed using JMP 8.0.2 software (SAS Institute).

\section{Results}

Frequency and Location of Postoperative CHB Sign

In all cases, bypass patency was confirmed with MR angiography. None of the cases presented the CHB sign in the preoperative study. Postoperative $\mathrm{CHB}$ signs in FLAIR images were detected in 112 surgically treated cases $(79.4 \%)$. Total CHB scores were 0 in 29 cases (20.6\%), 1 in 26 cases (18.4\%), 2 in 31 cases (22.0\%), 3 in 42 cases $(29.8 \%)$, and 4 in 13 cases $(9.2 \%)$. Neither acute cortical infarction (DWI positive) nor cortical hemorrhage was detected in this series. The CHB sign disappeared during the chronic period in all cases (Fig. 3). In the anterior part of the MCA territory, CHB scores were 0 in 66 


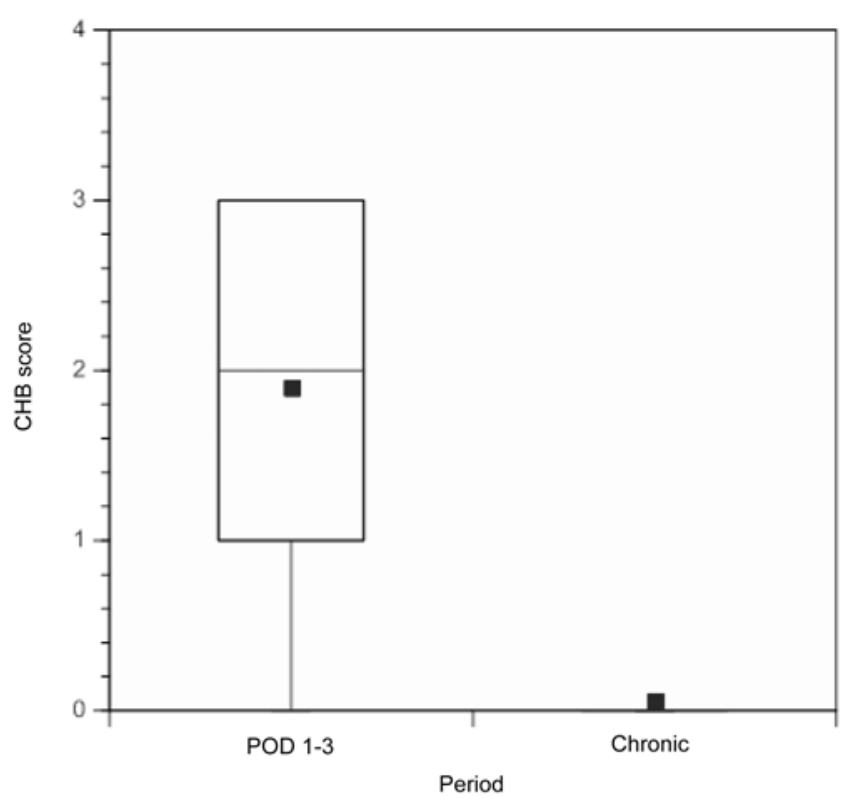

FIG. 3. The CHB scores obtained during the early PODs and during the chronic period are shown in the box plot.

cases (46.8\%), 1 in 59 (41.8\%), and 2 in 16 (11.3\%). In the posterior part, on the other hand, CHB scores were 0 in 34 cases (24.1\%), 1 in $39(27.7 \%)$, and 2 in $68(48.2 \%)$. The CHB sign was much more pronounced in the posterior part of the MCA territory (Fig. 4, p < 0.0001).

\section{The CHB Sign and Postoperative Symptoms}

Postoperative TNEs were observed in 86 surgically treated cases $(61.0 \%)$. Observed symptoms were numbness in 50 cases (35.5\%), aphasia in $36(25.5 \%)$, motor weakness in 17 (12.1\%), dysarthria in 15 (10.6\%), and other in $5(3.5 \%)$. The mean CHB scores of patients with and without TNEs were $2.31 \pm 0.13$ and $1.24 \pm 0.16$, respectively. Patients with TNEs showed significantly higher CHB scores than those without TNEs (Fig. 5, $\mathrm{p}<0.0001$ ). Furthermore, the total CHB score and duration of TNEs were correlated significantly $(\mathrm{p}<0.0001, \mathrm{r}=0.471)$.

\section{The CHB Sign and rCBF Increase}

The mean $\mathrm{rCBF}$ increase ratio was $1.12 \pm 0.21(0.68$ 1.73). One-way ANOVA revealed no significant relationship between CHB score and $\mathrm{rCBF}$ increase ratio $(\mathrm{p}=$ 0.775).

\section{Regional CBF Increase and TNEs}

Regional CBF increase ratios with and without TNEs were $1.15 \pm 0.033$ and $1.16 \pm 0.037$, respectively. No difference was evident between these 2 groups $(p=0.978)$. In addition, the $\mathrm{rCBF}$ increase ratio did not correlate with the duration of TNEs $(p=0.720, r=0.041)$.

\section{Discussion}

The CHB sign is defined in the present study as an "intraparenchymal" belt-like area of high signal intensity observed in the cortex in FLAIR images that differs from

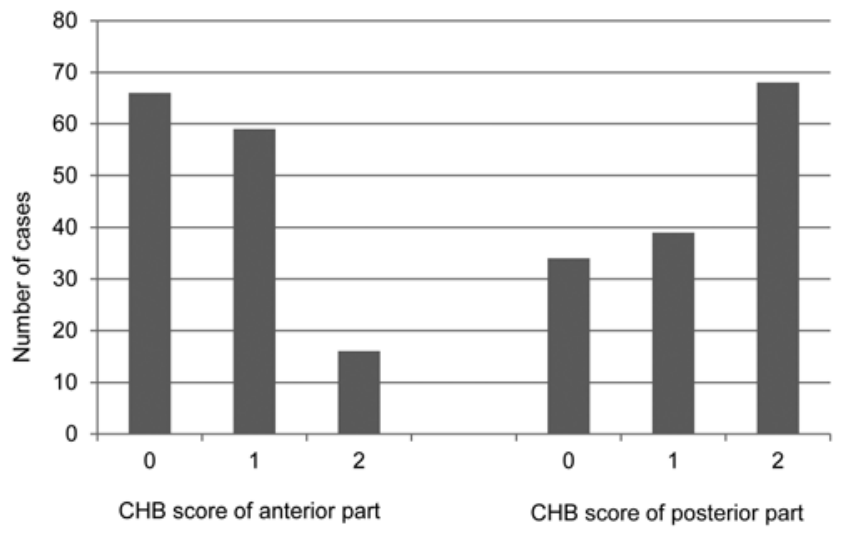

FIG. 4. The CHB scores of anterior and posterior parts. The CHB sign is more pronounced in the posterior part than in the anterior part of the MCA territory $(p<0.0001)$.

the original "ivy sign," the leptomeningeal area of high signal intensity observed in moyamoya disease. ${ }^{25}$ The ivy sign is thought to reflect dilated pial vasculature and slow leptomeningeal flow. ${ }^{16,25,29} \mathrm{Kaku}$ et al. used ${ }^{15} \mathrm{O}$-PET to reveal that a pronounced ivy sign has a positive correlation with increased cerebral blood volume (CBV) and a negative correlation with the $\mathrm{CBF} / \mathrm{CBV}$ ratio, which strongly supports this hypothesis. ${ }^{16}$ Although the ivy sign can accompany the postoperative CHB sign, they were carefully differentiated in this study. Whereas the ivy sign can be observed before surgery, ${ }^{11}$ CHB signs were detected only postoperatively. These findings after direct bypass for moyamoya disease have been known empirically by neurosurgeons, but their frequency and physiological meaning have been unclear. In the present study, the CHB sign appeared in $80.9 \%$ of the operative cases during the acute postoperative period, and total CHB scores showed significant correlation with the occurrence and duration of TNEs. Accordingly, a pronounced CHB sign during the early postoperative period can be a predictor of reversible postoperative neurological events in moyamoya disease.

On the other hand, the CHB sign did not correlate with an increase in rCBF. Interestingly, the CHB sign was ob-

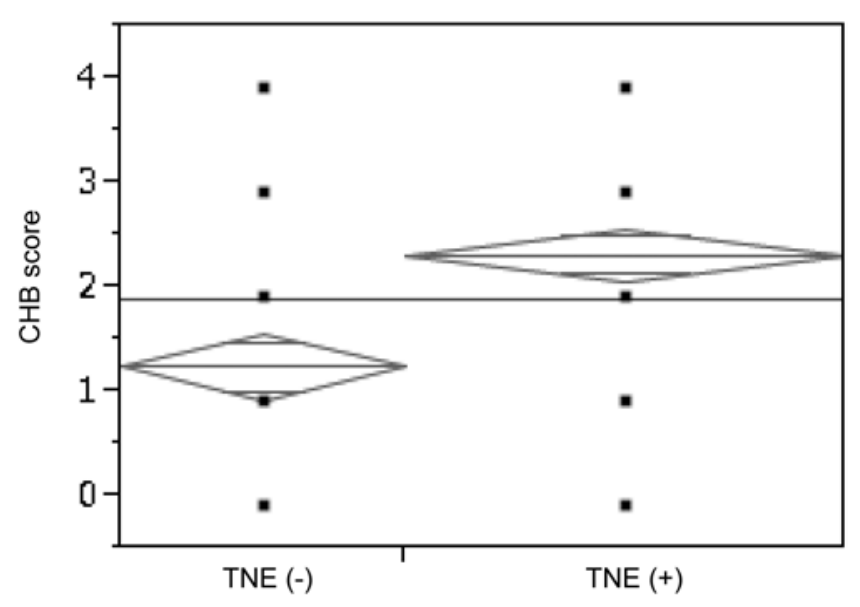

FIG. 5. The CHB score and postoperative TNEs. Patients with TNEs $(+)$ had significantly higher $\mathrm{CHB}$ scores than those without $(-)(p<0.0001)$. 
served more frequently in the posterior part of the MCA territory despite STA-MCA anastomosis having been performed in the anterior part $\left(\mathrm{M}_{4}\right.$ of frontal operculum). In other words, the CHB sign does not necessarily appear at the anastomosis site and is less related to the rCBF increase itself.

The pathophysiology of these TNEs has not been well elucidated. Since 2000, the transient local increase in $\mathrm{rCBF}$ after bypass surgery in moyamoya disease has been reported frequently, and some authors have attributed postoperative TNEs to this rCBF increase (local hyperperfusion). ${ }^{4,6,15,16,20,40}$ It has been reported, however, that cerebral infarction is the most frequent cause of postoperative complication after bypass surgery for moyamoya disease, surely resulting from "hypo"-perfusion. . $^{14,22,26,35-37}$ Moreover, the present study shows that the increase in rCBF ratio correlated neither with the occurrence nor the duration of TNEs. Therefore, it seems unreasonable to ascribe all transient neurological symptoms to local hyperperfusion phenomenon.

Horie et al. reported that a postoperative increase in the distinctness of the ivy sign in FLAIR images is a predictor of hyperperfusion..$^{11}$ As mentioned above, however, the original ivy sign is a leptomeningeal area of high signal intensity (extraparenchymal signal), suggesting slow flow in engorged pial arteries. ${ }^{25}$ Although it is unclear whether these authors adopted strict criteria for identifying the ivy sign, their report refers to "a linear high-signal intensity along the cortical sulci or brain surface" yet refers elsewhere to "the cortico-subcortical lesion," which suggests some ambiguity. In the present study, the CHB sign is carefully differentiated from the ivy sign and is confined to the intraparenchymal signal change.

Further study is needed to elucidate the pathological implications of the CHB sign. Because intensity shifts are transient and DWI never indicated high intensity, it can be speculated that the CHB sign is due to vasogenic edema and is very unlikely to reflect cytotoxic edema or infarction. Vasogenic brain edema is thought to be the result of autoregulatory failure leading to cerebral arteriolar dilation, breakdown of the blood-brain barrier, and extravasation of the fluid to the brain parenchyma. ${ }^{33}$ Cortical vasogenic edema has been reported in cases of posterior reversible encephalopathy syndrome (PRES) $)^{1,2,12}$ and hyperperfusion after carotid revascularization. ${ }^{13,19,39}$ In addition, in moyamoya disease, local hyperperfusion can cause cortical vasogenic edema. ${ }^{8}$

In the present study, however, the CHB sign is not correlated with the $\mathrm{rCBF}$ increase. The $\mathrm{CHB}$ sign does not seem to be the result of hyperperfusion, yet it is closely related to postoperative TNEs. Although the mechanism remains unclear, it could be hypothesized that preoperative vasodilation and hemodynamic change caused by direct revascularization could lead to some autoregulatory failure and extravasation of the fluid without a distinct increase in the rCBF. In PRES, it is postulated that the MRI abnormality may be due to the seizure activity itself rather than its cause. ${ }^{34}$ It is also possible, therefore, that some electrophysiological factors could be involved in the pathogenesis of the CHB sign and TNEs.

The curious location of the CHB sign might be a clue to its pathophysiology. The CHB sign was significantly pronounced in the posterior part of the MCA territory, far from the anastomotic point. Some disorders have been characterized by reversible vasogenic edema within specific locations in the brain. PRES, for example, usually shows vasogenic edema in the posterior parietal or temporo-occipital area. ${ }^{33}$ In iodinated contrast encephalopathy accompanying cortical vasogenic edema, occipital lobe symptoms are the most frequent. ${ }^{24}$ Although the factors determining the location of such lesions are poorly understood, it has been speculated that the cerebral vascular response can vary from site to site. Some researchers have reported that the posterior cerebral vessel has a relatively poor sympathetic innervation and is therefore more vulnerable to hemodynamic insults., $3,21,27$ Although further study is needed, such regional differences in vascular regulation might be a reason for the posterior tendency of the CHB sign.

The present study has some limitations. First, accurate inter- and intraobserver variability could not be calculated because the 2 neurosurgeons, who were blinded to the clinical conditions, made their determinations jointly, not independently. Because all slices of the FLAIR images were checked and a CHB score was calculated in each case, it is possible that some variability would have arisen if all judgments had been made independently.

Second, the difference in the degree of hemodynamic impairment between those patients with and those without the CHB sign could not be determined because of the lack of a highly quantitative evaluation. Our only available tool was ${ }^{123}$ I-IMP SPECT. In the pediatric cases, only the semiquantitative rCBF data (count activity) could be obtained because routine arterial blood sampling is difficult with these children. A less invasive method such as arterial spin labeling with MRI is a promising tool, but its validity for moyamoya disease has not been established. ${ }^{31}$

Third, the CHB score itself does not alter the postoperative clinical management at the present time. Careful research on the CHB sign, however, will provide important clues to the unknown mechanism of the frequent TNEs that affect patients with moyamoya disease. Postoperative routine MR study is required to detect the CHB sign, which may present a financial disadvantage. As mentioned above, however, it has been reported that perioperative ischemic stroke is the most frequent complication following bypass surgery for moyamoya disease. Because early detection and therapeutic intervention are mandatory, routine MR studies with FLAIR and DWI could be justified.

\section{Conclusions}

A pronounced CHB sign can be a predictor of TNEs after direct bypass surgery for moyamoya disease, but it is minimally related to the local hyperperfusion phenomenon. It is notable that the CHB sign is significantly pronounced in the posterior part of the MCA territory. Although it is speculated that this signal shift reflects vasogenic edema, further study is needed to reveal both the pathophysiology of the CHB sign and the mechanism of postoperative neurological events in moyamoya disease. 


\section{References}

1. Bartynski WS: Posterior reversible encephalopathy syndrome, part 1: fundamental imaging and clinical features. AJNR Am J Neuroradiol 29:1036-1042, 2008

2. Bartynski WS: Posterior reversible encephalopathy syndrome, part 2: controversies surrounding pathophysiology of vasogenic edema. AJNR Am J Neuroradiol 29:1043-1049, 2008

3. Dillon WP, Rowley H: The reversible posterior cerebral edema syndrome. AJNR Am J Neuroradiol 19:591, 1998

4. Fujimura M, Kaneta T, Mugikura S, Shimizu H, Tominaga $\mathrm{T}$ : Temporary neurologic deterioration due to cerebral hyperperfusion after superficial temporal artery-middle cerebral artery anastomosis in patients with adult-onset moyamoya disease. Surg Neurol 67:273-282, 2007

5. Fujimura M, Kaneta T, Shimizu H, Tominaga T: Symptomatic hyperperfusion after superficial temporal artery-middle cerebral artery anastomosis in a child with moyamoya disease. Childs Nerv Syst 23:1195-1198, 2007

6. Fujimura M, Shimizu H, Inoue T, Mugikura S, Saito A, Tominaga T: Significance of focal cerebral hyperperfusion as a cause of transient neurologic deterioration after extracranialintracranial bypass for moyamoya disease: comparative study with non-moyamoya patients using $\mathrm{N}$-isopropyl-p-[ $\left.{ }^{123} \mathrm{I}\right]$ iodoamphetamine single-photon emission computed tomography. Neurosurgery 68:957-965, 2011

7. Furuya K, Kawahara N, Morita A, Momose T, Aoki S, Kirino T: Focal hyperperfusion after superficial temporal arterymiddle cerebral artery anastomosis in a patient with moyamoya disease. Case report. J Neurosurg 100:128-132, 2004

8. Hayashi K, Horie N, Suyama K, Nagata I: Incidence and clinical features of symptomatic cerebral hyperperfusion syndrome after vascular reconstruction. World Neurosurg 78:447-454, 2012

9. Heros RC, Scott RM, Kistler JP, Ackerman RH, Conner ES: Temporary neurological deterioration after extracranialintracranial bypass. Neurosurgery 15:178-185, 1984

10. Higashi S, Matsuda H, Fujii H, Ito H, Yamashita J: Luxury perfusion syndrome confirmed by sequential studies of regional cerebral blood flow and volume after extracranial to intracranial bypass surgery: case report. Neurosurgery 25:85-89, 1989

11. Horie N, Morikawa M, Morofuji Y, Hiu T, Izumo T, Hayashi $\mathrm{K}$, et al: De novo ivy sign indicates postoperative hyperperfusion in moyamoya disease. Stroke 45:1488-1491, 2014

12. Hugonnet E, Da Ines D, Boby H, Claise B, Petitcolin V, Lannareix V, et al: Posterior reversible encephalopathy syndrome (PRES): features on CT and MR imaging. Diagn Interv Imaging 94:45-52, 2013

13. Ivens S, Gabriel S, Greenberg G, Friedman A, Shelef I: Blood-brain barrier breakdown as a novel mechanism underlying cerebral hyperperfusion syndrome. J Neurol 257:615620,2010

14. Iwama T, Hashimoto N, Yonekawa Y: The relevance of hemodynamic factors to perioperative ischemic complications in childhood moyamoya disease. Neurosurgery 38:11201126, 1996

15. Kaku Y, Iihara K, Nakajima N, Kataoka H, Fukuda K, Masuoka J, et al: Cerebral blood flow and metabolism of hyperperfusion after cerebral revascularization in patients with moyamoya disease. J Cereb Blood Flow Metab 32:20662075,2012

16. Kaku Y, Iihara K, Nakajima N, Kataoka H, Fukushima K, Iida $\mathrm{H}$, et al: The leptomeningeal ivy sign on fluid-attenuated inversion recovery images in moyamoya disease: positron emission tomography study. Cerebrovasc Dis 36:19-25, 2013

17. Karasawa J, Kikuchi H, Furuse S, Sakaki T, Yoshida Y: A surgical treatment of "moyamoya" disease "encephalo-myo synangiosis.” Neurol Med Chir (Tokyo) 17:29-37, 1977
18. Karasawa J, Touho H, Ohnishi H, Miyamoto S, Kikuchi H: Long-term follow-up study after extracranial-intracranial bypass surgery for anterior circulation ischemia in childhood moyamoya disease. J Neurosurg 77:84-89, 1992

19. Ketteler S, Djalali-Talab Y, Dafotakis M, Wiesmann M, Schulz JB, Haarmeier T: Teaching neuroimages: combined retinal and cerebral hyperperfusion syndrome after carotid thromboendarterectomy. Neurology 81:e166-e167, 2013

20. Kim JE, Oh CW, Kwon OK, Park SQ, Kim SE, Kim YK: Transient hyperperfusion after superficial temporal artery/ middle cerebral artery bypass surgery as a possible cause of postoperative transient neurological deterioration. Cerebrovasc Dis 25:580-586, 2008

21. Kur JK, Esdaile JM: Posterior reversible encephalopathy syndrome-an underrecognized manifestation of systemic lupus erythematosus. J Rheumatol 33:2178-2183, 2006

22. Kuroda S, Houkin K, Nunomura M, Abe H: Frontal lobe infarction due to hemodynamic change after surgical revascularization in moyamoya disease-two case reports. Neurol Med Chir (Tokyo) 40:315-320, 2000

23. Kuroda S, Kamiyama H, Abe H, Asaoka K, Mitsumori K: Temporary neurological deterioration caused by hyperperfusion after extracranial-intracranial bypass--case report and study of cerebral hemodynamics. Neurol Med Chir (Tokyo) 34:15-19, 1994

24. Leong S, Fanning NF: Persistent neurological deficit from iodinated contrast encephalopathy following intracranial aneurysm coiling. A case report and review of the literature. Interv Neuroradiol 18:33-41, 2012

25. Maeda M, Tsuchida C: "Ivy sign" on fluid-attenuated inversion-recovery images in childhood moyamoya disease. AJNR Am J Neuroradiol 20:1836-1838, 1999

26. Matsushima Y, Aoyagi M, Suzuki R, Tabata H, Ohno K: Perioperative complications of encephalo-duro-arterio-synangiosis: prevention and treatment. Surg Neurol 36:343-353, 1991

27. Meyer MA, Galloway G, Khan S: Transient blindness associated with reversible occipital white matter abnormalities: two patients studied by MR, CT, and ${ }^{18}$ F-FDG PET imaging. $\mathbf{J}$ Neuroimaging 8:240-242, 1998

28. Miyamoto S, Akiyama Y, Nagata I, Karasawa J, Nozaki K, Hashimoto N, et al: Long-term outcome after STA-MCA anastomosis for moyamoya disease. Neurosurg Focus 5(5):e5, 1998

29. Mori N, Mugikura S, Higano S, Kaneta T, Fujimura M, Umetsu A, et al: The leptomeningeal "ivy sign" on fluidattenuated inversion recovery MR imaging in Moyamoya disease: a sign of decreased cerebral vascular reserve? AJNR Am J Neuroradiol 30:930-935, 2009

30. Mugikura S, Takahashi S, Higano S, Shirane R, Kurihara N, Furuta S, et al: The relationship between cerebral infarction and angiographic characteristics in childhood moyamoya disease. AJNR Am J Neuroradiol 20:336-343, 1999

31. Noguchi T, Kawashima M, Irie H, Ootsuka T, Nishihara M, Matsushima T, et al: Arterial spin-labeling MR imaging in moyamoya disease compared with SPECT imaging. Eur J Radiol 80:e557-e562, 2011

32. Ohta T, Tanaka H, Kuroiwa T: Diffuse leptomeningeal enhancement, "ivy sign," in magnetic resonance images of moyamoya disease in childhood: case report. Neurosurgery 37:1009-1012, 1995

33. Ong B, Bergin P, Heffernan T, Stuckey S: Transient seizurerelated MRI abnormalities. J Neuroimaging 19:301-310, 2009

34. Raghavendra S, Ashalatha R, Krishnamoorthy T, Kesavadas C, Thomas SV, Radhakrishnan K: Reversible periictal MRI abnormalities: clinical correlates and long-term outcome in 12 patients. Epilepsy Res 73:129-136, 2007

35. Sakamoto T, Kawaguchi M, Kurehara K, Kitaguchi K, Fu- 
ruya $\mathrm{H}$, Karasawa J: Postoperative neurological deterioration following the revascularization surgery in children with moyamoya disease. J Neurosurg Anesthesiol 10:37-41, 1998

36. Sakamoto T, Kawaguchi M, Kurehara K, Kitaguchi K, Furuya $\mathrm{H}$, Karasawa J: Risk factors for neurologic deterioration after revascularization surgery in patients with moyamoya disease. Anesth Analg 85:1060-1065, 1997

37. Sato K, Shirane R, Yoshimoto T: Perioperative factors related to the development of ischemic complications in patients with moyamoya disease. Childs Nerv Syst 13:68-72, 1997

38. Uno M, Nakajima N, Nishi K, Shinno K, Nagahiro S: Hyperperfusion syndrome after extracranial-intracranial bypass in a patient with moyamoya disease-case report. Neurol Med Chir (Tokyo) 38:420-424, 1998

39. van Mook WNKA, Rennenberg RJMW, Schurink GW, van Oostenbrugge RJ, Mess WH, Hofman PAM, et al: Cerebral hyperperfusion syndrome. Lancet Neurol 4:877-888, 2005

40. Zhao WG, Luo Q, Jia JB, Yu JL: Cerebral hyperperfusion syndrome after revascularization surgery in patients with moyamoya disease. Br J Neurosurg 27:321-325, 2013

\section{Disclosures}

Dr. Iihara received a grant from Nihon Medi-Physics Co., Ltd.

\section{Author Contributions}

Conception and design: Hamano. Acquisition of data: Hamano, Kataoka. Analysis and interpretation of data: Hamano, Kataoka, Morita. Drafting the article: Hamano. Critically revising the article: Takahashi. Reviewed submitted version of manuscript: Takahashi. Statistical analysis: Hamano. Administrative/techni$\mathrm{cal} /$ material support: Morita, Maruyama, Satow, Iihara. Study supervision: Kataoka.

\section{Supplemental Information}

\section{Previous Presentations}

Portions of this work were presented in abstract and oral forms at the 42nd Annual Meeting of the Japanese Society on Surgery for Cerebral Stroke, Tokyo, Japan, March 22, 2013, and at the 25th Annual Meeting of the Japanese Society of Cerebral Blood Flow and Metabolism, Sapporo, Japan, November 2, 2013; and in abstract and poster forms at the 72nd Annual Meeting of the Japan Neurosurgical Society, Kanagawa, Japan, October 16, 2013.

\section{Correspondence}

Jun C. Takahashi, Department of Neurosurgery, National Cerebral and Cardiovascular Center, 5-7-1, Fujishirodai, Suita City, Osaka 565-8565, Japan. email: juntak@ncvc.go.jp. 